\title{
A study of the analgesic efficacy and safety of use of paracetomol formulations after third molar surgery
}

\author{
Pain control with paracetamol from a sustained release formulation and a standard release formulation after third \\ molar surgery: a randomised controlled trial by P Coulthard, C M Hill, J W Frame, H Barry, B D Ridge, and T H Bacon \\ Br Dent J 2001; 191: 319-324
}

\section{Objective}

To compare the analgesic efficacy and safety of a sustained release (SR) paracetamol formulation (Panadol Extend) with a standard immediate release (IR) formulation (Panadol) after third molar surgery.

\section{Design}

A multi-centre, double-blind, randomised clinical trial.

\section{Methods \\ Patients received either a single oral dose of SR paracetamol or IR paracetamol for pain after the removal of at least one impacted third molar requiring bone removal under general anaesthesia. Post-operative pain and pain relief assessments were undertaken at time intervals up to 8 hours. Global assessments of effectiveness were made at 4 and 8 hours. Any adverse events were also recorded.}

\section{Results \\ Of 627 randomised patients, 314 were treated with SR paracetamol and 313 with IR paracetamol. In the per protocol population at 4 hours, $35.1 \%$ of the 252 patients on SR paracetamol rated the study medication as very good or excellent compared with $27.7 \%$ of the 258 patients on IR paracetamol. There were few statistically significant differences among the secondary parameters but where they did occur they favoured SR paracetamol. Trends in favour of SR paracetamol were observed among the secondary parameters and these tended to emerge at the later time points. For example, while there was no statistically significant}

difference in time to re-medication between the treatment groups, the estimated time to re-medication was longer for patients treated with SR paracetamol ( $4 \mathrm{hr} 5 \mathrm{~min}$ ) compared with IR paracetamol ( $3 \mathrm{hr} 10 \mathrm{~min})$. The high rate of re-medication observed is consistent with that reported for IR paracetamol using the post-operative dental pain model ${ }^{4,6}$. No difference was observed between the SR paracetamol and IR paracetamol treatment groups in distribution, incidence or severity of adverse events.

\section{Conclusions}

SR paracetamol and IR paracetamol are clinically and statistically equivalent. While SR paracetamol and IR paracetamol were similar in terms of both onset of analgesia and peak analgesic effect, SR paracetamol had a longer duration of activity than IR paracetamol. The safety profiles of SR paracetamol and IR paracetamol were found to be very similar.

In Brief

- A sustained release paracetamol formulation and a standard immediate release paracetamol formulation are clinically and statistically equivalent.

- While SR paracetamol and IR paracetamol are similar in terms of both development of analgesia and peak analgesic effect, SR paracetamol has a longer duration of activity than IR paracetamol.

- The safety profiles of SR paracetamol and IR paracetamol are very similar.

\begin{abstract}
Comment
The control of post-operative pain after 1 dental surgical procedures remains the main reason for prescribing analgesics in dental practice. The present study evaluates single doses of two paracetamol preparations in a large cohort of patients with pain after third molar surgery. Their results showed that essentially there was no difference between the two preparations as assessed by a variety of primary and secondary outcome measures.

Does this finding come as a surprise and will it change the prescribing habits of general dental practitioners?

The study was well conducted but was based upon the hypothesis that differences in the pharmacokinetics of the two paracetamol preparations would have some impact on the drug's efficacy, especially the duration of action. It would also suggest that paracetamol pharmacokinetics are important determinants of the
\end{abstract}

drug's efficacy, a point which has been difficult to establish. ${ }^{1}$ Relating pharmacokinetics to efficacy, irrespective of the drug, is a challenge for both pharmacologists and clinicians. Yet, this very hypothesis forms the basis of many drug developments as the pharmaceutical industry modifies existing preparations to improve efficacy, onset and duration of action. It is often the case, as exemplified by the present study, that such developments fail to impact upon the clinical arena.

What impact will the findings from this study have on general dental practice? Probably very little. There is a wealth of information supporting the efficacy of nonsteroidal anti-inflammatory drugs in post-operative dental pain. Analgesics such as ibuprofen and aspirin will remain the drugs of choice to treat this painful condition. Paracetamol would be an alternative in those patients where an NSAID cannot be prescribed (eg asthmatics or active peptic ulceration).

In summary, this is a well conducted clinical trial, which logistically would have taken a huge effort to organise. It is disappointing that the results are weak with respect to comparative efficacy of the two compounds. There is unlikely to be a wholesale switch to paracetamol as the analgesic of choice for post-operative dental pain.

\section{R. A. Seymour}

Head of the Department of Restorative Dentistry, The Dental School, University of Newcastle upon Tyne, Framlington Place, Newcastle upon Tyne

1 Seymour R A, Rawlins M D. Pharmacokinetics of parenteral paracetamol and its analgesic effects in post-operative dental pain. Eur J Clin Pharmacol 1981; 20: 215-218. 